\title{
Illustrating orientation changes of the insertion trajectory during cochlear implant electrode array insertion
}

M. Geraldine Zuniga, Department of Otolaryngology and Cluster of Excellence EXC 2177/1 "Hearing4all", Hannover Medical School, Hanover, Germany, ZunigaManrique.Maria@mh-hannover.de

Georg Böttcher, Department of Otolaryngology, Hannover Medical School, Hanover, Germany, boettcher.georg@mhhannover.de

Viktor Schell, Department of Otolaryngology, Hannover Medical School, Hanover, Germany, schell.viktor@mhhannover.de

Thomas Lenarz, Department of Otolaryngology and Cluster of Excellence EXC 2177/1 “Hearing4all”, Hannover Medical School, Hanover, Germany, lenarz.thomas@mh-hannover.de

Thomas S. Rau, Department of Otolaryngology and Cluster of Excellence EXC 2177/1 "Hearing4all”, Hannover Medical School, Hanover, Germany, rau.thomas@mh-hannover.de

\section{Introduction}

Recent investigations focused on the optimization of atraumatic cochlear implant surgery have highlighted the relevance of the electrode array (EA) insertion trajectory. This is particularly studied in the context of minimally-invasive "keyhole" and robotic-assisted approaches, e.g. to avoid injuring structures inside and outside the cochlea. However, little is known about the natural, manual movements and trajectory followed during the insertion process. The present work illustrates the orientation changes within the trajectory a surgeon follows during insertions of EAs into a human cadaveric cochlea.

\section{Methods}

An EA insertion tool equipped with a gyroscope was developed in our laboratory. During the insertion trials, the gyroscope captures the tool's spatial orientation. A human head specimen and a single EA were used to perform insertions into a cochlea. A cochlear implant surgeon performed all insertion trials. The recorded orientations were compared to the initial orientation upon cochlea entry to assess the surgeon's range of motion by calculating the angle between orientation vectors.

\section{Results}

Fifteen EA insertions were performed with an average maximal deviation from the initial orientation of $10.6^{\circ}\left(\mathrm{SD} 6.1^{\circ}\right)$ across trials. The largest orientation changes were seen towards the last ten seconds of each insertion trial. A negative relationship between degree of axis change and number of insertion trial was observed $(\mathrm{r}=-0.5)$.

\section{Conclusion}

Manual EA insertions into a cadaveric cochlea revealed an insertion trajectory with maximum orientation changes of approximately $10^{\circ}$ degrees. The observed trend on decreasing range of motion with increasing number of insertion trials may be attributed to EA deformation and softening but other contributing factors need to be further elucidated with several EAs. Future evaluations can help determine if this orientation change is influenced by surgeon expertise. 


\section{A New Tool for Measuring Intracochlear Contact Forces during Electrode Insertion}

Rolf Salcher, (Department of Otolaryngology and Cluster of Excellence "Hearing4All”, Hannover Medical School), Hannover, Germany, Salcher.Rolf@mh-hannover.de

Martina Nullmeier, (Cochlear Limited), Sydney, Australia, mnullmeier@cochlear.com

Jakob Cramer, (Department of Otolaryngology and Cluster of Excellence "Hearing4All", Hannover Medical School), Hannover, Germany, and (Cochlear Limited), Sydney, Australia, Cramer.Jakob@mh-hannover.de

Nick Pawsey, (Cochlear Limited), Sydney, Australia, npawsey@ cochlear.com

Thomas Lenarz, (Department of Otolaryngology and Cluster of Excellence "Hearing4All", Hannover Medical School), Hannover, Germany, Lenarz.Thomas@mh-hannover.de

Thomas S. Rau, (Department of Otolaryngology and Cluster of Excellence "Hearing4All", Hannover Medical School), Hannover, Germany, Rau.Thomas@mh-hannover.de

\section{Introduction}

Insertion force measurements are a standard method for characterizing cochlear implant electrode arrays as it is assumed that insertion force is correlated to trauma. The insertion forces are often measured in a simplified artificial cochlea model (ACM) which is mounted on a force sensor. This allows the summation of the forces opposing the insertion to be measured, but not the distribution of contact forces on specific areas of the cochlea. To investigate this spatial distribution of forces we developed the segmented cochlea insertion force measurement system.

\section{Methods}

The core of the setup is a 2D-ACM which is divided into four segments, each connected to a separate 3D force sensor with $2 \mathrm{~N}$ capacity. To enable realistic friction conditions the model is immersed into a lubricant bath (here: saline solution). The electrode is moved by a linear stage. Custom-made software for motion control and data acquisition using three universal analog input modules was developed. For initial insertion tests two slim straight and two slim modiolar electrode arrays have been used.

\section{Results}

Twenty insertions were performed. Summed force profiles compared with uniaxial forces measured in a single-piece model show no artefacts due to gaps between the segments. Force analysis showed opposing forces acting on different segments of the cochlea, particularly perpendicular to the direction of insertion, which would not be measurable in a single-piece model.

Slim Modiolar caused significantly lower insertion forces than slim straight.

\section{Conclusion}

This setup enables investigation of the forces acting on different parts of the cochlea during insertion. In contrast, with standard ACMs and a single sensor only net forces can be measured, which might lead to wrong conclusions. The setup is a promising tool for further investigation of different lateral wall and perimodiolar electrodes and their typical contact force magnitudes and patterns of distribution. 


\section{A method for image-guided positioning of cochlear specimens in insertion test benchs using 3D printed stands.}

Thomas S. Rau, Department of Otolaryngology and Cluster of Excellence EXC 2177/1 "Hearing4all”, Hannover Medical School, Hanover, Germany, rau.thomas@mh-hannover.de

Jakob Cramer, Department of Otolaryngology, Hannover Medical School, Hanover, Germany, cramer.jakob@mhhannover.de

M. Geraldine Zuniga, Department of Otolaryngology and Cluster of Excellence EXC 2177/1 "Hearing4all", Hannover Medical School, Hanover, Germany, ZunigaManrique.Maria@mh-hannover.de

Georg Böttcher, Department of Otolaryngology, Hannover Medical School, Hanover, Germany, boettcher.georg@mhhannover.de

Thomas Lenarz, Department of Otolaryngology and Cluster of Excellence EXC 2177/1 "Hearing4all”, Hannover Medical School, Hanover, Germany, lenarz.thomas@mh-hannover.de

\section{Introduction}

Cochlear implant configuration includes a receiver-stimulator and an electrode array (EA). The EA is the portion inserted into the cochlea. Insertion tests using artificial cochlear models (ACM) or ex vivo specimens are widely used methods during EA development to characterize EA design properties, including insertion forces. Measured forces are directly linked to the orientation of the cochlear lumen with respect to the insertion axis of the test bench. While desired insertion directions can be predefined in ACM experiments by design, specimens are individually shaped and the cochlear lumen is embedded invisibly. Therefore a new method for accurate, individual positioning with respect to a previously planned trajectory is required.

\section{Methods}

Key element of the proposed method is a customizable pose setting adapter (PSA) used to adjust the specimen's fine positioning. After rigid fixation of the specimen to a holder featuring spherical registration markers and a subsequent cone beam computer tomography an insertion trajectory is planned. Planning data is used to calculate the individual shape of thePSA. Finally the PSA is 3D printed and mounted between force sensor and specimen holder to align the planned trajectory with the test bench's insertion axis.

\section{Results}

All necessary hard- and software have been developed which include the specimen holder, a software for registration and trajectory planning, and a custom Matlab script whose output drives a parametric CAD file of the PSA. Usability of image-guided specimen alignment was demonstrated in initial experiments using a porcine cochlea specimen.

\section{Conclusion}

The presented stereotactic positioning procedure enables accurate alignment of the cochlear lumen to the insertion axis. This enables higher repeatability in ex vivo insertion experiments due to precise, image-guided control of the insertion direction and its systematic investigation as an important parameter affecting insertion forces. 


\section{Cochlear implants as in vivo oxygen sensors}

Andreas Weltin, Jochen Kieninger, Gerald A. Urban, IMTEK - Department of Microsystems Engineering, University of Freiburg, Freiburg, Germany, andreas.weltin@imtek.uni-freiburg.de, kieninger@imtek.uni-freiburg.de, gerald.urban@imtek.uni-freiburg.de

Susan Arndt, Nicole Rosskothen-Kuhl, Department of Otorhinolaryngology, University Medical Center Freiburg, Freiburg, Germany, susan.arndt@uniklinik-freiburg.de,nicole.rosskothen-kuhl@uniklinik-freiburg.de

\section{Introduction}

The cochlear implant (CI) is a widely used neuroprothesis that activates the auditory nerve by direct electrical stimulation and thus compensates hearing loss in patients. The platinum electrodes of the CI are comparable to those of micro bio- and chemo-sensors. Using the CI electrodes as sensors opens access to the microenvironment of the implant and the state of the electrode itself, promising novel insight into implant biocompatibility, function, and long-term stability.

\section{Methods}

We developed an electrochemical method to turn CI electrodes into electrochemical dissolved oxygen sensors using a chronoamperometric protocol that measures oxygen via reduction at the Pt electrode. CI electrodes were used as working and counter electrodes, and a silver/silver chloride wire was the reference electrode. All electrodes of a three channel implant (MED-EL, Austria) were completely inserted through a cochleostomy in medio-dorsal direction into the middle turn of the left cochlea of anesthetized adult rats, while the reference electrode was placed in the middle ear of the same side.

\section{Results}

We demonstrated stable, reproducible, and highly linear sensor performances in vitro, using dissolved oxygen, but also hydrogen peroxide, as test substances. In acute experiments in vivo, we obtained a stable baseline signal, indicating a well-oxygenated environment. Supplying the animals with pure oxygen to the nose led to a fast and reproducible increase in intracochlear oxygen levels and return to baseline. Electrical intracochlear stimulation via the same CI electrodes resulted in well-differentiated electrically evoked auditory brainstem responses, not affected by previous electrochemical measurements.

\section{Conclusion}

We demonstrated that CI electrodes can be turned into chemical microsensors in vivo using appropriate electrochemical methods. These can also be combined directly with methods for long-term in situ corrosion monitoring. Besides the microenvironment, this can give access to electrode degradation investigating stimulation protocols and ultimately improve electrode stability and function by implant-life-long monitoring. 


\section{Digital Live Imaging of intraoperative electrocochleography - hearing preservation during Cochlear Implantation}

Diana Arweiler-Harbeck ${ }^{1}$, diana.arweiler-harbeck@uk-essen.de, Victoria D’heygere ${ }^{1}$, Victoria.d’heygere@uk-essen.de, Moritz Meyer ${ }^{1}$, Moritz.meyer@uk-essen.de, Stefan Hans ${ }^{1}$, Stefan.hans@uk-essen.de, Laura Waschkies ${ }^{1}$, Laura.waschkies@uk-essen.de, Kristina Anton ${ }^{2}$, kanton@cochlear.com, Horst Hessel ${ }^{2}$, hhessel@cochlear.com, Armin Schneider ${ }^{3}$, aschneider@munichimaging.de, Thomas Heiler ${ }^{3}$, theiler@munichimaging.de, Benedikt Höing ${ }^{1}$, benedikt.hoeing@uk-essen.de

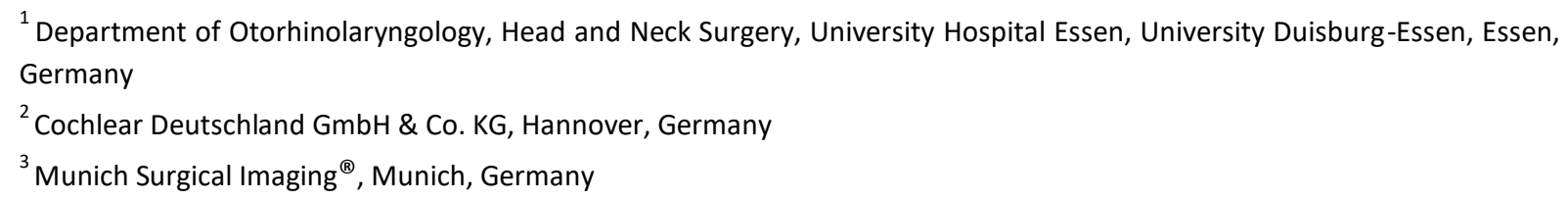

\section{Introduction:}

Intraoperative electrocochleography (ECochG) during cochlear implantation is a promising tool to preserve residual hearing. However, the time gap between insertion of the electrode and acoustic feedback from the audiologist to the surgeon causes delay and subsequently irreparable damage to cochlear structures. For the first time real-time visualization of intraoperative ECoch $G$ via digital microscope (ARRISCOPE ${ }^{\circledR}$ ) directly to the surgeon using "picture-in-picture mode" was successfully performed.

\section{Methods:}

Intraoperative setting was preliminarily tested in the lab. 15 patients with residual hearing underwent cochlear implantation. Intraoperative Ecoch G responses were collected and direct visualization during the time of insertion into the surgeon's field of view in the binoculars using augmented realtime digital imaging was realized. Time of electrode insertion was taken. Hearing preservation was determined by testing postoperative behavioral thresholds.

\section{Results:}

Digital live visualization of intraoperative ECochG using image augmentation in a digital microscope (ARRISCOPE ${ }^{\circledR}$ ) was successfully performed and enabled direct adaptation of the surgeon's insertion behavior. Direct visualization via PiP led to significantly longer insertion times compared to not directly visualized Ecochg measurements. Postoperative behavioral thresholds were comparable to preoperatively taken thresholds. Preservation of residual hearing was possible.

\section{Discussion:}

This study is the first to describe digital visualization of intraoperative EcochG as a new method enabling the surgeon to directly react to changes in amplitude of the cochlea microphonics. Our results show that augmentation of the intraoperative live imaging with electrical potentials can add to hearing preservation during cochlear implantation. 


\section{A method to determine the accuracy of shape setting of thin, spirally shaped Nitinol wires}

Tim Ehmann, Department of Otolaryngology, Hannover Medical School, Hanover, Germany, ehmann.tim@mhhannover.de

M. Geraldine Zuniga, Department of Otolaryngology and Cluster of Excellence EXC 2177/1 "Hearing4all", Hannover Medical School, Hanover, Germany, zunigamanrique.maria@mh-hannover.de.

Thomas Lenarz, Department of Otolaryngology and Cluster of Excellence EXC 2177/1 "Hearing4all”, Hannover Medical School, Hanover, Germany, lenarz.thomas@mh-hannover.de

Thomas S. Rau, Department of Otolaryngology and Cluster of Excellence EXC 2177/1 "Hearing4all”, Hannover Medical School, Hanover, Germany, rau.thomas@mh-hannover.de

\section{Introduction}

Electric stimulation of the auditory nerve using a cochlear implant (CI) is presumed to be superior when the electrode array is placed close to the inner wall of the cochlea. Nitinol, a commonly used shape memory alloy and famous for its temperatue-induced shape change is explored to be utilised as an actuator that enables an intracochlear shape change of the EA from a straight configuration (necessary for the insertion) to a spiral shape fitting to the inner wall. Shape setting of the thin Nitinol wires is crucial; therefore a method to determine its accuracy was required and developed to quantify the quality of the shape setting.

\section{Methods}

To measure the actual shape of the delicate, thin Nitinol wires $(\varnothing 100 \mu \mathrm{m})$ a contactless, optical method was developed. For each wire a photomicrograph of the final shape was captured and processed using a custom Matlab algorithm. Threshold based segmentation followed by morphological operations to remove artefacts were applied to extract the wire's shape. Utilizing an iterative closest point (ICP) algorithm the actual shape was registered to the desired spiral path. After registration, the root mean squared error was calculated as a measure for the "shape error" - the deviation between both spirals. In total 147 Nitinol wires of 16 batches with different transformation temperatures were analysed to quantify the reliability of the shape-setting procedure.

\section{Results}

Optical determination of the spiral shape was successfully applied in all 147 samples. Average shape error was $0.06 \pm$ $0.02 \mathrm{~mm}$. Deviation from the desired shape was $<0.1 \mathrm{~mm}(0.15 \mathrm{~mm})$ in $95 \%(99 \%)$ of the samples.

\section{Conclusion}

The presented method is suitable to control the trained shape of thin Nitinol wires. Our results confirm a high reliability of the shape-setting used in thin Nitinol actuators intended for future applications in CI electrode arrays. 